\title{
Guest editorial: a new era of ATL and HTLV-1 research
}

\author{
Masao Matsuoka
}

Received: 31 August 2011/Revised: 17 September 2011/Accepted: 20 September 2011/Published online: 4 October 2011

(c) The Japanese Society of Hematology 2011

In the mid 1970s, Dr. Uchiyama, Dr. Yodoi and Dr. Takatsuki noticed that the frequency of T-cell leukemia in Japan was higher than that in Western countries. A novel finding was that patients with T-cell leukemia had similar clinical features: frequent skin lesions, hypercalcemia, the presence of characteristic leukemic cells in the peripheral blood, and opportunistic infections. Based on these clinical observations, they proposed this disease as a distinct clinical entity, adult T-cell leukemia (ATL). Surprisingly, the birthplaces of ATL patients were concentrated in Kyushu, suggesting that this disease was caused by a specific pathogen. In 1980, Dr. Gallo's group at NIH found the first human retrovirus, now called human T-cell leukemia virus, from a patient with Mycosis fungoides. In 1981, Dr. Hinuma's group reported that ATL patients had antibodies to an ATL cell line, indicating that ATL was caused by a pathogen. Thereafter, Dr. Yoshida revealed the sequence of the virus in ATL cells, finally proving that HTLV is a causative agent of ATL. HTLV is the first human retrovirus discovered to cause a human disease. Later, it was reported that HTLV-1 induced inflammatory diseases in addition to ATL. It goes without saying that the discovery of HTLV-1 has had tremendous impacts on medicine, virology and human society.

Thereafter, Dr. Uchiyama moved to NIH as a postdoctoral fellow in Dr. Waldmann's laboratory, and started to generate monoclonal antibodies to $\mathrm{T}$ cells. Quite by accident, he used cultured $\mathrm{T}$ cells from the same patient from whom Dr. Gallo's group established a cell line and identified HTLV. He succeeded in generating a monoclonal antibody to activated $\mathrm{T}$ cells, anti-Tac. Later, this antibody

M. Matsuoka ( $)$

Institute for Virus Research, Kyoto University,

53 Shogoin Kawara-cho, Kyoto 606-8507, Japan

e-mail:mmatsuok@virus.kyoto-u.ac.jp was found to react to the IL-2 receptor alpha chain, which is abundantly expressed on HTLV-1 infected cells. This was a great serendipity. Thus, research on HTLV-1 and ATL has made a great contribution to not only virology, but also immunology and hematology.

However, many problems remain. First, the frequency of HTLV-1 infection does not appear to be decreasing as rapidly as expected. Dr. Watanabe reports on the recent status of HTLV-1 infection in Japan and the world. It was speculated that the number of HTLV-1 infected individuals would decrease rapidly. However, recent studies show that the number of HTLV-1 carriers remains unchanged in Japan, and rather has increased in the Tokyo and Kansai areas. These findings require us to pay attention to HTLV-1 infection in the non-endemic areas of Japan.

Second, the pathogenesis of ATL is still not fully understood. Previous studies suggested the significance of Tax in the leukemogenesis of ATL. However, Tax expression is frequently disrupted in ATL cases. The HTLV-1 bZIP factor (HBZ) gene is expressed in all ATL cases, and promotes the proliferation of ATL cells. HBZ expression converts cells to regulatory T cells. Dr. Yasunaga and I summarize recent progress on the understanding of HTLV-1, especially HBZ.

Finally and most importantly, new therapies are needed for ATL, a fatal disease. Stem cell transplantation can cure some ATL patients. However, many ATL patients cannot receive this treatment since the disease is not controllable or the donors are not found. Dr. Ishida and Dr. Ueda reported the development of an anti-CCR4 monoclonal antibody as a novel therapeutic agent for ATL. This new agent will be a lamp of hope for ATL patients. Thirty-five years after the discovery of ATL, a new era of research on HTLV-1 and ATL has finally arrived. 